\title{
MiR-378 Plays an Important Role in the Differentiation of Bovine Preadipocytes
}

\author{
Si-Yuan Liu ${ }^{a}$ Yang-Yang Zhang ${ }^{a}$ Yan Gao Lian-Jiang Zhang ${ }^{a, b}$ Hong-Yan Chen ${ }^{a}$ \\ Qian Zhou ${ }^{a}$ Meng-Long Chaic Qing-Ying Li $^{a}$ Hao Jiang ${ }^{a}$ Bao Yuan ${ }^{a}$ \\ Li-Sheng Dai ${ }^{a}$ Jia-Bao Zhang ${ }^{a}$ \\ ${ }^{a}$ College of Animal Sciences, Jilin University, Changchun Jilin, ${ }^{b}$ College of Animal Science and \\ Technology, Jilin Agricultural Science and Technology College, Jilin Jilin, 'College of Animal Science and \\ Technology, Jilin Agricultural University, Changchun Jilin, China
}

\section{Key Words}

Bovine $•$ miR-378 • E2F2 • RANBP10 • Preadipocyte differentiation

\begin{abstract}
Background: Adipocyte, the main cellular component of white adipose tissue, plays a vital role in energy balance in higher eukaryotes. In recent years, adipocytes have also been identified as a major endocrine organ involved in immunological responses, vascular diseases, and appetite regulation. In farm animals, fat content and categories are closely correlated with meat quality. MicroRNAs (miRNAs), a class of endogenous single-stranded non-coding RNA molecules, participate in the regulation of adipocyte differentiation and adipogenesis through regulating the transcription or translation of target mRNAs. MiR-378 plays an important role in a number of biological processes, including cell growth, cell differentiation, tumor cell survival and angiogenesis. Methods: In the present study, bioinformatics analysis and dual-luciferase reporter assay were used to identify and validate the target genes of miR-378. In vitro cell transfection, quantitative reverse transcription polymerase chain reaction (RT-qPCR), western blot analysis, Oil Red $\mathrm{O}$ staining, and triglyceride content measurement were conducted to analyze the effects of miR-378 on bovine preadipocyte differentiation. Results: MiR-378 was induced during adipocyte differentiation. In the differentiated adipocytes overexpressing miR378 , the volume of lipid droplets was enlarged, and the triglyceride content was increased. Moreover, the mRNA expression levels of the adipocyte differentiation marker genes, peroxisome proliferator-activated receptor gamma (PPAR $\gamma)$ and sterol regulatory elementbinding protein (SREBP), were significantly elevated in the differentiated, mature adipocytes. In contrast, the mRNA expression level of preadipocyte factor 1 (Pref- 1 ) was markedly reduced. E2F transcription factor 2 (E2F2) and Ras-related nuclear (RAN)-binding protein 10 (RANBP10) were the two target genes of miR-378. The mRNA expression levels of E2F2 and RANBP10 did not significantly change in bovine preadipocytes overexpressing miR-378. However, the protein expression levels of E2F2 and RANBP10 were markedly reduced. Conclusion: MiR-378
\end{abstract}

S.-Y. Liu and Y.-Y. Zhang contributed equally to this article.

Jia-Bao Zhang

and Li-Sheng Dai 
promoted the differentiation of bovine preadipocytes. E2F2 and RANBP10 were the two target genes of miR-378, and might involve in the effects of miR-378 on the bovine preadipocyte differentiation.

Copyright $\odot 2015$ S. Karger AG, Basel

\section{Introduction}

Adipose tissue regulates the body's energy balance by functioning as the main energy reserve during caloric excess and facilitating fat mobilization during food deprivation. In recent years, adipocytes have also been identified as a major endocrine organ involved in immunological responses, vascular diseases, and appetite regulation [1]. In farm animals, fat content and categories are closely correlated with meat quality. The maintenance of the functions of adipose tissue depends on adipocytes, the main cellular component of white adipose tissue. Currently, the studies on adipocytes are mainly focused on human and mouse adipocytes, while little research has been conducted on bovine adipocytes. Adipocytes are derived from pluripotent stem cells, which differentiate first into lipoblasts, then into preadipocytes and eventually into mature adipocytes $[2,3]$. The differentiation of preadipocytes toward mature adipocytes is regulated by a variety of differentiation-related transcription factors and is accompanied by the temporal expression of a series of adipogenic genes and synthase genes related in lipid metabolism [1]. Peroxisome proliferator-activated receptor $\gamma$ (PPAR $\gamma$ ), sterol-regulatory element binding proteins (SREBPs) and preadipocyte factor 1 (Pref-1) are now recognized as the adipocyte differentiation marker genes. PPAR $\gamma$ and SREBP expression levels increase gradually during adipocytes differentiation, whereas Pref- 1 expression level gradually decreases [4, 5]. In addition, the process of adipocyte differentiation is regulated by multiple signaling pathways and microRNAs (miRNAs).

MiRNAs are a class of endogenous single-stranded non-coding RNA molecules that are approximately 20 nucleotides (nt) in length [6]. Studies have shown that miRNAs regulate the transcription or translation of the target gene mRNAs [7], thus participating in the regulation of adipocyte differentiation and adipogenesis [8-10]. Kajimoto et al. monitored the expression levels of 80 miRNAs during 3T3-L1 cell line differentiation and found that the expression levels of 21 miRNAs exhibit dynamic changes [9]. MiR-143 exhibits a significant promoting effect on adipocyte differentiation [11]. The miR-27 gene family specifically inhibits adipocyte formation through suppressing PPAR $\gamma$ and CCAAT-enhancer-binding protein $\alpha(\mathrm{C} / \mathrm{EBP} \alpha)[8,12]$. In addition, let-7 and miR-130 exert a negative regulatory effect on adipocyte differentiation [13], whereas miR-103 promotes adipocyte differentiation and regulates insulin secretion[14]. A previous study has shown that miR-378 exerts a promoting effect on the differentiation of the 3T3-L1 cell line [15]. However, the mechanism by which miR-378 regulates primary preadipocyte differentiation remains unclear.

To understand the regulatory effect of miR-378 on the differentiation of bovine preadipocytes, we analyzed the expression pattern of miR-378 in the process of bovine preadipocyte differentiation. To further investigate the regulatory role of miR-378, preadipocytes were transfected with miR-378 mimics and cultured under differentiationinducing conditions. Moreover, the target genes of miR-378 were identified using bioinformatics and an in vitro luciferase reporter assay. The changes in the morphology of preadipocytes and various physiological indices were examined. The results of the present study would provide a basis for further investigation of miRNA-mediated physiological regulation of adipose tissue in bovine.

\section{Materials and Methods}

Isolation, in vitro culture and differentiation induction of primary bovine preadipocytes

Healthy calves ( 1 day of age) were selected for this study. Experiments were performed in accordance with the Guiding principles in the use of animals, adopted by the Chinese Association for Laboratory 
Animal Sciences. The study plan was approved by the Ethics Committee on the Use and Care of Animals, Jilin University. The abdominal cavity of the calf was opened aseptically and the mesentery was removed. The mesentery was washed several times with phosphate buffered saline (PBS) containing 6\% penicillin/ streptomycin (PPA Laboratories) and then transported to the cell culture room. The mesenteric adipose tissue was separated from the connective tissue. Blocks of adipose tissue were placed into centrifuge tubes and cut into pieces. After addition of $5 \mathrm{ml} \mathrm{0.2 \%} \mathrm{collagenase} \mathrm{I} \mathrm{(Sigma,} \mathrm{USA),} \mathrm{the} \mathrm{centrifuge} \mathrm{tubes} \mathrm{were} \mathrm{sealed}$ and placed into a $37^{\circ} \mathrm{C}$ water bath for $1.5 \mathrm{~h}$ to allow sufficient digestion of the adipose tissue. The cell suspension obtained after collagenase digestion was filtered through 80-mesh and 200-mesh cell sieves. The resulting filtrate was centrifuged at $1500 \mathrm{r} / \mathrm{min}$ for $10 \mathrm{~min}$, and the supernatant was discarded. Bovine preadipocytes in the pellets were cultured in DMEM/F12 complete medium (Gibco, USA) containing 10\% fetal bovine serum (Gibco, USA) and 1\% penicillin/streptomycin under standard conditions of $37^{\circ} \mathrm{C}$ and $5 \% \mathrm{CO}_{2}$. After the cells were grown to $100 \%$ confluence, the cultured medium was replaced with induction medium consisting of DMEM/F12, $10 \mu \mathrm{g} / \mathrm{ml}$ insulin (Sigma, USA), $33 \mu \mathrm{M}$ calcium pantothenate (Sigma, USA), $17 \mu \mathrm{M}$ bovine transferrin (Sigma, USA), 10\% fetal bovine serum and 1\% penicillin/streptomycin to induce differentiation (day 0 ). The medium was changed every three days.

Prediction of target genes and luciferase reporter assays

Using miRNA target gene prediction programs, such as TargetScan (http://www.targetscan.org/ mmu_50/) and RNA22 (http://cbcsrv.watson.ibm.com/rna22.html), the putative miR-378 binding sites at the 3'-untranslated region (UTR) of potential miR-378 target genes in bovine were analyzed, and candidate target genes were selected. Based on the seed region sequences of the candidate target genes provided by the National Center for Biotechnology Information (NCBI), primers targeting the gene segments containing the seed sequences were designed with Xho I and Not I restriction enzyme sites incorporated. In addition, mutagenic primers were designed to specifically mutate $3-5$ bases in the seed region of the candidate target genes. The primer sequences are listed in Table 1. After primer annealing, candidate target sequences containing wild-type and mutant seed regions were synthesized. The wild-type and mutant sequences were cloned into psiCHECK- 2 to construct wild type and mutant reporter plasmids, respectively.

MiR-378 mimics (5 pmol) or negative control (NC; 5 pmol) miR (Shanghai GenePharma Co., Ltd., China) of cattle were cotransfected with 2 ng of the candidate target gene-containing wild type, mutant or empty psiCHECK-2 vector (negative control) into the NIH3T3 mouse embryonic fibroblast cell line. After $48 \mathrm{~h}$ of culture, Renilla luciferase and firefly luciferase activities were measured using a chemiluminescence reader.

Table 1. Primers of targeting the seed region of the candidate target genes

\begin{tabular}{ll}
\hline Primer Name & Primer Sequence (5'-3') \\
\hline E2F2-WT-F & TCGAGCCGGGAATGAATCTTATGGCGAAGTCCAGCCGC \\
E2F2-WT-R & GGCCGCGGCTGGACTTCGCCATAAGATTCATTCCCGGC \\
RANBP10-WT-F & TCGAGGAGCCCCTGTCTTGCTGATAGTCCAGGCGC \\
RANBP10-WT-R & GGCCGCGCCTGGACTATCAGCAAGACAGGGGCTCC \\
E2F2-MU-F & TCGAGCCGGGAATGAATCTTATGGCGAACAGGAGCCGC \\
E2F2-MU-R & GGCCGCGGCTCCTGTTCGCCATAAGATTCATTCCCGGC \\
RANBP10-MU-F & TCGAGGAGCCCCTGTCTTGGACTTACAGGAGGCGC \\
RANBP10-MU-R & GGCCGCGCCTCCTGTAAGTCCAAGACAGGGGCTCC \\
\hline
\end{tabular}

Table 2. MiR-378 mimics / NC sequence

\begin{tabular}{|c|c|}
\hline Name & Sequence (5'-3') \\
\hline miR-378 mimics & $\begin{array}{l}\text { sense ACUGGACUUGGAGUCAGAAGGC } \\
\text { antisense CUUCUGACUCCAAGUCCAGUUU }\end{array}$ \\
\hline $\mathrm{NC}$ & $\begin{array}{l}\text { sense UUCUCCGAACGUGUCACGUTT } \\
\text { antisense ACGUGACACGUUCGGAGAATT }\end{array}$ \\
\hline
\end{tabular}


Table 3. Primers used for RT-qPCR analysis

\begin{tabular}{ll}
\hline Name & Sequence $\left(5^{\prime}-3^{\prime}\right)$ \\
\hline miR-378-RT & GTC GTA ATC CAG TGC GTGTCGTGGAGTCGGCAATTGCACTGGATACGACGCCTTCT \\
miR-378-F & GGGACTGGACTTGGAGTCA \\
miR-378-R & GTGCGTGTCGTGGAGTCG \\
U6-RT & CGCTTCACGAATTTGCGTGTCAT \\
U6-F & CGCTTCACGAATTTGCGTGTCAT \\
U6-R & GCTTCGGCAGCACATATACTAAAAT \\
E2F2-F & GACGTACCAGGACATCCGTGC \\
E2F2-R & GTGATCCCAGGGTCGCTGTT \\
RANBP10-F & TTCCCACACGCACAACAC \\
RANBP10-R & TCACTGGTCTCCTGGTTACTG \\
PPAR $\gamma-\mathrm{F}$ & GTGAAGTTCAACGCACTGGA \\
PPAR $\gamma-\mathrm{R}$ & ATGTCCTCAATGGGCTTCAC \\
SREBP-F & CCACAACGCCATCGAGAAACGCTAC \\
SREBP-R & GGCCTTCCCTGACCNCCCAACTTAG \\
Pref-1-F & AAGCACCGGCAGACAAGA \\
Pref-1-R & CAGAGGAGGGAGTCGTCAGTA \\
GAPDH-F & AATGAAAGGGCCATCACCATC \\
GAPDH-R & GTGGTTCACGCCCATCACA \\
\hline
\end{tabular}

Transfection of bovine preadipocytes

Bovine preadipocytes were seeded into 12 -well plates at a density of $1 \times 10^{6}$ cells/well in DMEM/F12 complete culture medium ( $1 \mathrm{ml}$ of medium per well). Once the cells were grown to $50-70 \%$ confluence, the complete medium was removed and replaced with $800 \mu \mathrm{l}$ of Opti-MEM medium (Gibco, USA). After 2 h, 2 $\mu \mathrm{l}$ of Lipofectamine ${ }^{\mathrm{TM}} 2000$ (Invitrogen, USA) and $4 \mu \mathrm{l}$ of miR-378 mimics (or $4 \mu \mathrm{l}$ of NC) were each added separately to $100 \mu \mathrm{l}$ of 0 pti-MEM medium and incubated at room temperature for $5 \mathrm{~min}$. The LipofectamineOpti-MEM and miR-378/NC-Opti-MEM, were combined and incubated at room temperature for $20 \mathrm{~min}$. The $200 \mu \mathrm{lmiR}-378 / \mathrm{NC}$-Lipofectamine mixture was added into the culture plates. After $6 \mathrm{~h}$ of culture, medium containing miR-378/NC-Lipofectamine was replaced with DMEM/F12 complete medium. Subsequent experiments were then conducted. The sequences of the miR-378 mimics and NC are shown in Table 2.

RNA extraction and quantitative real-time reverse transcription polymerase chain reaction (RT-qPCR)

Total cellular RNA was extracted using Trizol reagent (Invitrogen, USA) in accordance with the instruction manual provided by the manufacturer. Reverse transcription of miR-378 was conducted using the ReverTra AceR qPCR RT kit (TOYOBO, Japan). One $\mu$ g of RNA was first denatured by heating at $65^{\circ} \mathrm{C}$ for $5 \mathrm{~min}$. Subsequently, the reverse transcription reaction was performed after addition of the denatured RNA to the following reaction system: $2.0 \mu \mathrm{l}$ of $5 \times \mathrm{RT}$ buffer, $0.5 \mu \mathrm{l}$ of miR-378 RT primer $(10 \mu \mathrm{M}), 0.5 \mu \mathrm{l}$ of U6 RT primer $(10 \mu \mathrm{M}), 0.5 \mu \mathrm{l}$ of RT enzyme mix, and RNase-free $\mathrm{ddH}_{2} \mathrm{O}$ up to $10.0 \mu \mathrm{l}$, with the following condition: reverse-transcription at $37^{\circ} \mathrm{C}$ for $15 \mathrm{~min}$ and enzyme inactivation at $98^{\circ} \mathrm{C}$ for $5 \mathrm{~min}$. The reaction products were stored in $\mathrm{a}-80^{\circ} \mathrm{C}$ freezer.

RT-qPCR was performed using SYBR Premier Dimer Eraser ${ }^{\mathrm{TM}}$ (TaKaRa, Japan) and cDNA as the template according to the instruction manual provided by the manufacturer. The reaction system was as follows: $0.5 \mu \mathrm{l}(10 \mathrm{pmol} / \mathrm{L})$ of upstream primer, $0.5 \mu \mathrm{l}(10 \mathrm{pmol} / \mathrm{L})$ of downstream primer, $8.0 \mu \mathrm{l}$ of $\mathrm{ddH}_{2} \mathrm{O}$, $1 \mu \mathrm{l}$ of template and $10 \mu \mathrm{l}$ of SYBR ${ }^{\mathrm{R}}$ Premix Ex Taq II $(2 \mathrm{x})$, with the following conditions: $95^{\circ} \mathrm{C}$ for $30 \mathrm{~s}$; and 40 cycles of $95^{\circ} \mathrm{C}$ for $5 \mathrm{~s}$ and $60{ }^{\circ} \mathrm{C}$ for $30 \mathrm{~s}$. The U6 small nuclear RNA (snRNA) was used as an internal control for miR-378, and glyceraldehyde 3-phosphate dehydrogenase (GAPDH) was used as an internal control for other genes. Experimental data were analyzed using a relative quantification method $\left(2^{-\triangle \Delta C T}\right)$. All experiments were repeated three times. The RT-qPCR primers are described in detail in Table 3.

Determination of triglyceride content and Oil Red 0 staining

After adipocyte differentiation, the culture medium was discarded. The triglyceride content in adipocytes was determined using the Triglycerides Enzymatic Assay Kit E1013 (APPLYGEN, China) in accordance with the instruction manual of the kit. For Oil Red 0 staining, the cells were washed three times with PBS and fixed in 10\% neutral buffered formalin for $30 \mathrm{~min}$. The cells were then washed twice with 
PBS, stained with 10\% Oil Red 0 solution (solvent: 70\% ethanol) for 15 min, decolorized rapidly in 75\% ethanol, and washed twice with PBS. Subsequently, the cells were counterstained with hematoxylin for $15 \mathrm{~s}$ and washed three times with PBS. The stained cells were observed under a microscope and photographed $[16,17]$.

\section{Western blot analysis}

The cells were rinsed with PBS, treated with $0.25 \%$ trypsin (Gibco, USA) for 2 min, harvested, and transferred to centrifuge tubes. Cellular proteins were extracted using the Total Protein Extraction Kit (Sangon biotech, China) and the protein concentration was measured. Then $50 \mathrm{ug}$ of protein samples were mixed evenly with $5 \times$ sample loading buffer, denatured at $98^{\circ} \mathrm{C}$ for $15 \mathrm{~min}$, separated by sodium dodecyl sulfate polyacrylamide gel electrophoresis (SDS-PAGE) on a $12 \%$ gel and transferred to a membrane. The membrane was blocked for $2 \mathrm{~h}$ and then incubated with primary antibodies that were diluted with blocking buffer (E2F2 rabbit polyclonal IgG, Ras-related nuclear (RAN)-binding protein 10 (RANBP10) rabbit polyclonal IgG or $\beta$-actin mouse monoclonal IgG1; $1: 1000$ dilution) for $12 \mathrm{~h}$ at $4^{\circ} \mathrm{C}$ on a shaker. The membrane was then incubated with corresponding secondary antibodies (goat anti-rabbit IgG-HRP or goat anti-mouse IgG-HRP; 1:2000 dilution) at room temperature (RT) for $1 \mathrm{~h}$. All the antibodies were purchased from Santa Cruz Biotechnology, Inc. (USA). The target protein bands were imaged using the Tanon-5200 automated chemiluminescence analyzer (Tanon Science and Technology Co., Ltd., China).

\section{Statistical analysis}

Experimental data were analyzed using the analysis of variance (ANOVA) module of the SPSS16.0 statistical software. The data are expressed as the means \pm standard error (SE).

\section{Results}

Isolation, in vitro culture of primary bovine preadipocytes

After $24 \mathrm{~h}$ of culture, a portion of the isolated cells adhered to the culture surface and assumed a spindle-like shape or an irregular triangular shape. As the culture continued, the cells gradually became long, spindle-shaped and plump (Fig. 1A and 1B). After induction, a large number of cells differentiated and gradually filled with lipid droplets (Fig. 1C and 1D). In addition, the mRNA expression levels of the PPAR $\gamma$, SREBP and Pref- 1 marker genes were measured before and after adipocyte differentiation, and significant differences were detected. The mRNA expression levels of PPAR $\gamma$ and SREBP were elevated, but the mRNA expression level of Pref- 1 was reduced (Fig. 1E). These results indicated that the isolated bovine preadipocytes were satisfactory to be used in the subsequent experiments.

To study the expression pattern of miR-378 in the process of bovine preadipocyte differentiation, RT-qPCR was conducted to examine the expression levels of miR-378 at different stages of cell differentiation. The results showed that as the degree of adipocyte differentiation increased, the expression levels of miR-378 became gradually elevated. The miR-378 expression level increased significantly at $12 \mathrm{~d}$ after induction of differentiation and was approximately 2 -fold higher than the level at $0 \mathrm{~d}$ (Fig. 1F). Since the expression level of miR-378 significantly correlated with the differentiation process, we speculated that miR378 might be involved in the regulation of bovine preadipocyte differentiation.

\section{The effect of miR-378 on bovine preadipocyte differentiation}

To investigate whether miR-378 plays a role in bovine preadipocyte differentiation, miR378 was overexpressed in bovine preadipocytes. MiR-378 mimics and NC were transfected into bovine preadipocytes. The miR-378 expression level was significantly upregulated in the miR-378 mimics group compared with the NC group $(\mathrm{p}<0.01)$. These results indicated the successful overexpression of miR-378 in bovine preadipocytes (Fig. 2A).

Differentiation of bovine preadipocyte was induced in the cells transfected with miR-378 mimics and NC. The triglyceride contents and expression levels of the adipocyte differentiation marker genes (PPAR $\gamma$, SREBP and Pref-1) in the differentiated adipocytes were examined. After $12 \mathrm{~d}$ of differentiation, cells were stained with Oil Red 0 . The number 


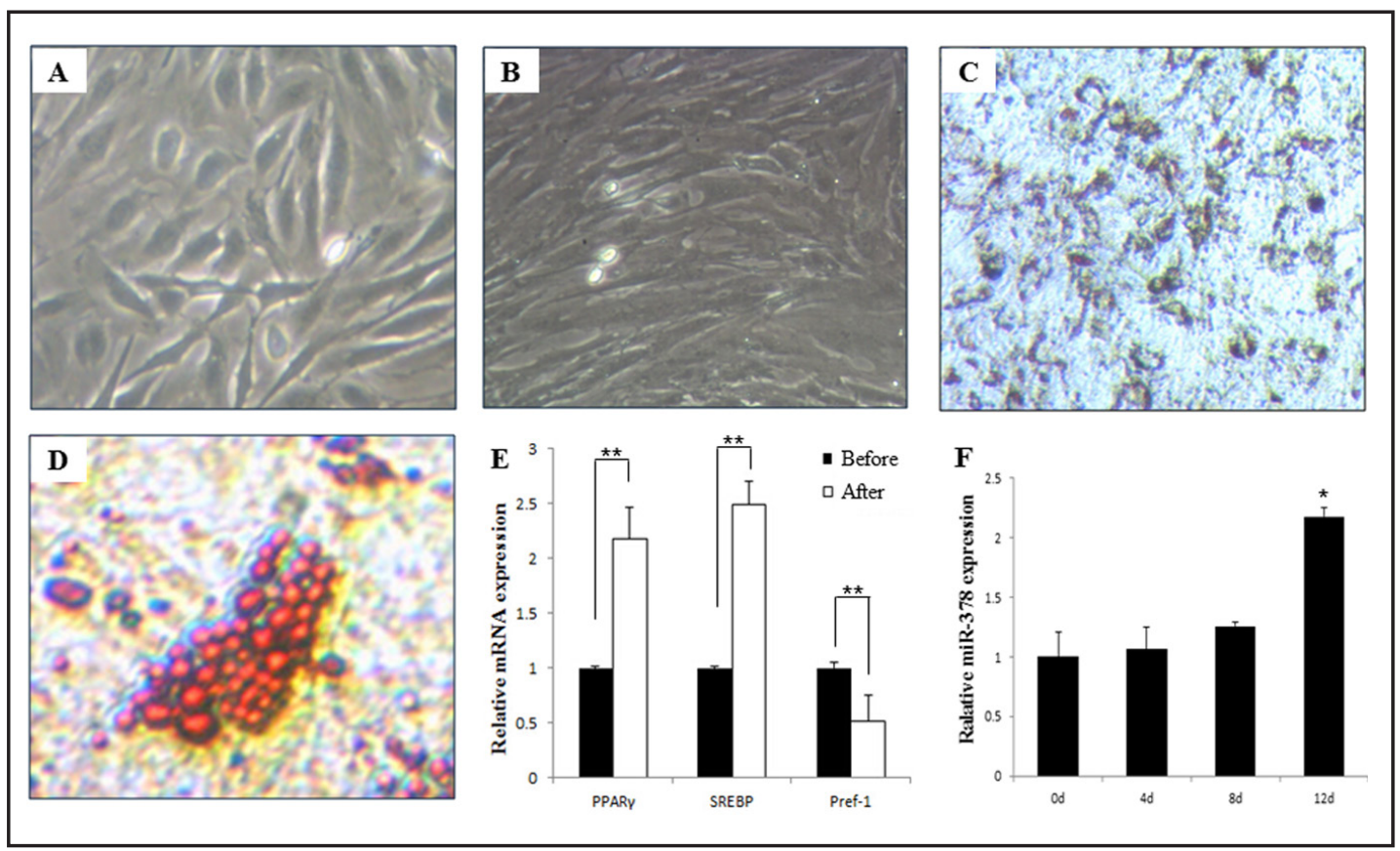

Fig. 1. The isolation, in vitro culture, differentiation induction, and changes in the mRNA expression of preadipocyte genes of bovine preadipocytes. A: Bovine preadipocytes adhered to the culture surface and assumed a spindle-like shape or an irregular triangular shape $(100 \times)$. B: Before differentiation, the bovine preadipocytes gradually became long, spindle-shaped and plump $(100 \times)$. C, Dorphological changes and lipid droplet formation in bovine preadipocytes after inducing differentiation (oil red 0 staining, $100 \times, 400$ $\times$ ). E: Changes in the mRNA expression levels of the PPAR $\gamma$, SREBP and Pref-1 genes after inducing differentiation. The mRNA from uninduced preadipocytes were used the control and the GAPDH gene was used as an internal reference. F: Changes of the miR-378 expression levels during the adipocyte differentiation process. Uninduced preadipocytes were used as the control and U6 was used as an internal reference. Data are expressed as means \pm SE. All experiments were repeated three times. ${ }^{*} \mathrm{p}<0.05,{ }^{* *} \mathrm{p}<0.01$.

and volume of lipid droplets were increased in the group of cells transfected with miR-378 mimics compared to the NC group (Fig. $2 \mathrm{~B}$ and $2 \mathrm{C}$ ). In addition, the triglyceride content was significantly higher in the miR-378 mimics group compared to the NC group $(\mathrm{p}<0.05)$ (Fig. 2D). Moreover, the mRNA expression levels of PPAR $\gamma$ and SREBP were significantly elevated in the miR-378 mimics group compared to the NC group $(\mathrm{p}<0.05)$, whereas the expression level of Pref- 1 mRNA was markedly decreased $(\mathrm{p}<0.05)$ (Fig. 2E). These data suggested that miR-378 promoted the differentiation of bovine preadipocytes.

\section{Prediction and validation of miR-378 target genes}

MiRNAs exert their biological functions by regulating the transcription or translation of the target gene mRNAs [7]. To analyze the regulatory mechanism of miR-378, target genes of miR-378 were predicted by bioinformatics and validated by luciferase reporter assays. Based on prediction scores in different programs, several candidates were selected for validating. It was indicated in comparison with cells transfected with $\mathrm{NC}$ that the relative luciferase activity (Renilla/Firefly) in the presence of miR-378 mimics significantly decreased $(p<0.01)$ in the cells cotransfected with E2F2 or RANBP10 wild-type constructs (Fig. 3A and 3B). Therefore, the two candidate target genes of miR-378, E2F2 and RANBP10, were selected for further analysis. As shown in the schematic diagram of the secondary structure formed between miR-378 and its target sequences, miR-378 could bind tightly to the 3'-UTR of E2F2 and RANBP10 in bovine (Fig. 3C). To further validate miR-378 responsive element in E2F2 and RANBP10 mRNA, mutated constructs were prepared at the sequence complementary to miR378 seed region (Fig. 3D). Dual luciferase reporter assay demonstrated the relative luciferase 


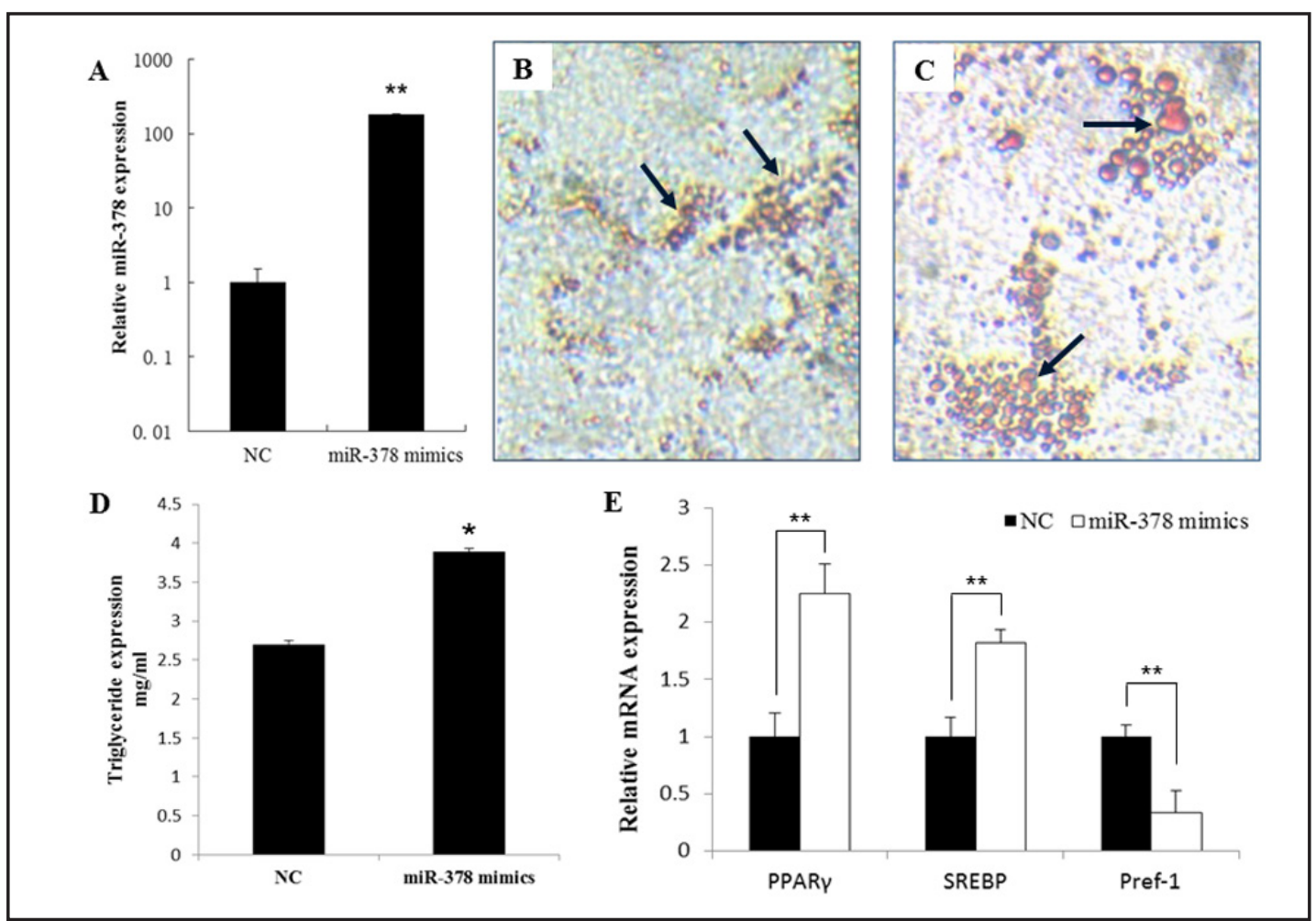

Fig. 2. Overexpression of miR-378 affected bovine preadipocyte differentiation and regulated mRNA expression levels of PPAR $\gamma$, SREBP and Pref- 1 genes. A: Relative expression levels of miR-378 in the NC and miR-378 mimics groups. The miR-378 expression level was significantly upregulated in the miR-378 mimics group compared with the NC group. B: Lipid droplet formation in the NC group after differentiation induction (oil red $\mathrm{O}$ staining, $400 \times$ ). The arrow indicates a differentiated adipocyte. C: Lipid droplet formation in the miR-378 mimics group after differentiation induction (oil red 0 staining, $400 \times$ ). The arrow indicates a differentiated adipocyte. There were significantly more and bigger lipid droplets in the miR-378 mimics group (C) than in the NC group (B). D: Triglyceride content measurement. E: The effects of miR-378 overexpression on the mRNA expression levels of PPAR $\gamma$, SREBP and Pref- 1 genes. Compared with the NC group, the miR-378 mimics group exhibited significantly increased mRNA expression levels of PPAR $\gamma$, SREBP , and decreased mRNA expression levels of Pref-1. The experiments were repeated three times. Data are expressed as the means $\pm \mathrm{SE},{ }^{*} \mathrm{p}<0.05,{ }^{* *} \mathrm{p}<0.01$.

activity in the miRNA response element (MRE) mutated group completely restored even in the presence of a miR-378 mimics when compared to the wild-type group $(\mathrm{p}<0.05$; Fig. $3 \mathrm{~A}$ and $3 \mathrm{~B}$ ). Taken together, the data suggested that miR-378 could target E2F2 and RANBP10 mRNA directly by binding the MRE within the 3'-UTR.

Targeted regulation of endogenous E2F2 and RANBP10 in bovine preadipocytes by miR378

To further investigate the mechanism by which miR-378 targets and regulates E2F2 and RANBP10, the effect of miR-378 overexpression on the mRNA and protein expression levels of endogenous E2F2 and RANBP10 was examined. Bovine preadipocytes were transfected with miR-378 mimics and NC. The RT-qPCR results showed that miR-378 failed to significantly affect the mRNA expression levels of E2F2 and RANBP10 (Fig. 4A and 4B). However, western blot analysis showed that the protein expression levels of E2F2 and RANBP10 were markedly reduced in the miR-378 mimics group in comparison to the NC group (Fig. 4C and 4D). These results indicated that the regulatory effect of miR-378 on its target genes, E2F2 and RANBP10, was achieved through inhibiting mRNA translation.

\section{KARGER}




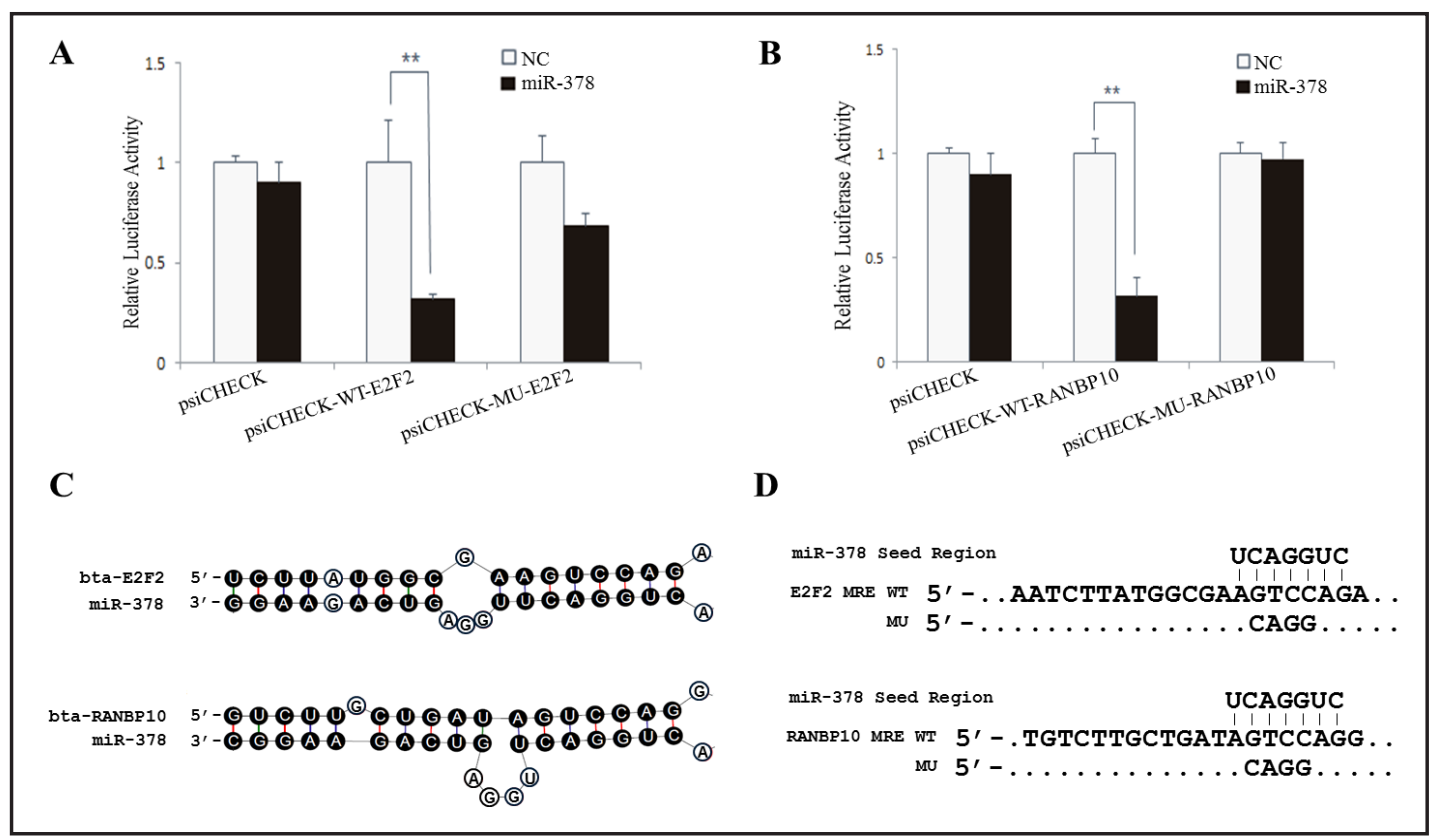

Fig. 3. Analysis of miR-378 responsive elements in the E2F2 and RANBP10 mRNA. A: Dual luciferase assays with E2F2 MRE wild-type (WT) (psiCHECK-WT-E2F2) or mutant (MU) (psiCHECK-MU-E2F2) constructs in the presence of miR-378 mimics or NC. B: Dual luciferase assays with RANBP10 MRE WT (psiCHECK-WT-RANBP10) or MU (psiCHECK-MU-RANBP10) constructs in the presence of miR-378 mimics or NC. C: Duplex structure between miR-378 and MRE in the E2F2 and RANBP10 mRNA predicted by mfold program (http://mfold.rna.albany.edu/?q=mfold). D: Diagrams of WT or mutant MU constructs of miR-378 MRE in the E2F2 and RANBP10 mRNA. Four nucleotides mutations were generated in the E2F2 and RANBP10 binding sites to miR-378 seed region using psiCHECK-2. Data are indicated as the means \pm SE derived from triplicate transfectants of three independent experiments. ${ }^{* *} \mathrm{p}<0.01$.

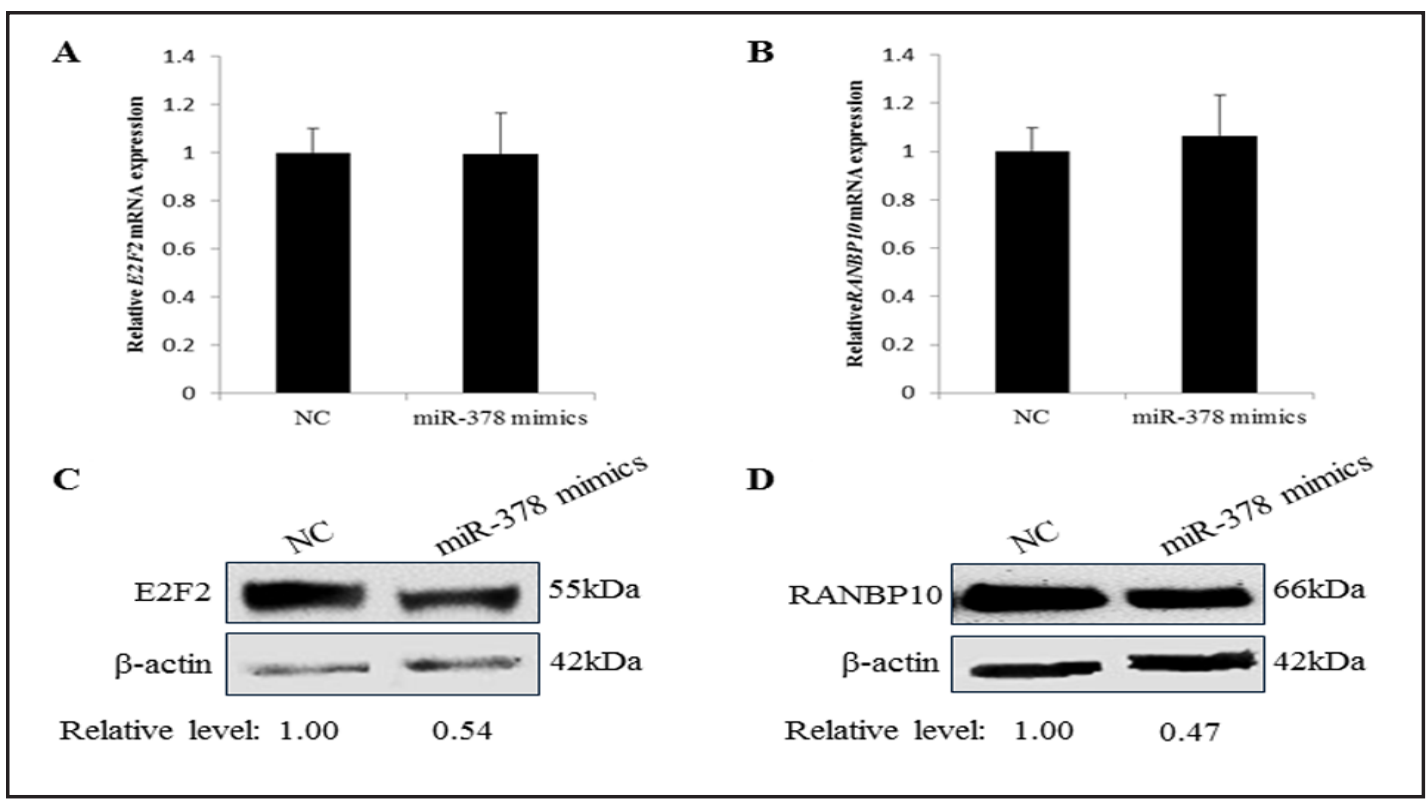

Fig. 4. MiR-378 represses E2F2 and RANBP10 protein expression with minor effect on mRNA level. A,B: Quantitative RT-qPCR measurements of E2F2 and RANBP10 mRNA from NC or miR-378 mimics transfected bovine preadipocytes. C,D: Western blot analysis of E2F2 and RANBP10 protein expression levels in the transfected bovine preadipocytes. The $\beta$-actin protein was used as an internal reference. Results presented are from three independent studies. Data are presented as the means \pm SE. 


\section{Discussion}

MiRNAs are widely expressed in mammalian tissues and organs involved in the regulation of many important biological processes. Studies have shown that miR-378 is closely associated with cell growth, cell differentiation, tumor cell survival and angiogenesis [18-20]. MiR-378 regulates tumor cell proliferation through targeting sulfatase 1 (SULF1), sex determining region Y (SRY)-box 7 (SOX7) and insulin-like growth factor 1 (IGF1) [2022]. In addition, miR-378 can also regulate cell differentiation. Gagan et al. found that miR-378 indirectly downregulates the myogenic inhibitory factor, MyoR, through targeted regulation of MyoD, thereby promoting the differentiation of myoblasts in C2C12 mouse cell line [18]. Xu et al. demonstrated that miR-378 inhibits the secretion of aromatase during porcine folliculogenesis, thus affecting the reproductive traits of pigs [23]. Adipose tissue is not merely a passive organ of energy storage as it is also an endocrine organ capable of secreting a variety of hormone-like substances $[24,25]$. Maintenance of the functions of adipose tissue mainly depends on the differentiation capacity of adipocytes and the degree of adipocyte differentiation in the organism[1]. It has been reported that miR-378 exerts a certain regulatory effect on the differentiation of 3T3-L1 cell line [15]. Jin et al. analyzed the correlation among 89 miRNAs and six different traits of cattle[26]. Among all the miRNAs examined, miR-378 exhibited the most significant correlation with the backfat thickness in cattle.

In the present study, we successfully isolated and cultured primary bovine preadipocytes and analyzed the expression pattern of miR-378 in the differentiation process of bovine preadipocytes. The results showed that the miR-378 expression levels gradually increased during the differentiation process and that the expression of miR-378 was significantly elevated at the late stage of differentiation. Therefore, we speculated that miR-378 exerted a certain regulatory effect on the differentiation of bovine preadipocytes.

To investigate whether miR-378 plays a role in bovine preadipocyte differentiation, we overexpressed miR-378 in bovine preadipocytes and then induced preadipocyte differentiation. After induction of differentiation, the volume of lipid droplets was increased in mature adipocytes overexpressing miR-378. In addition, the triglyceride contents were elevated, and the mRNA expression levels of the adipocyte differentiation marker genes, PPAR $\gamma$ and SREBP, were significantly increased. In contrast, the Pref-1 mRNA expression level was markedly reduced in mature adipocytes overexpressing miR-378. Studies have shown that as early markers of adipocyte differentiation, PPAR $\gamma$ and SREBP can be detected in preadipocytes. After induction of differentiation, the expression of PPAR $\gamma$ and SREBP increases rapidly and reaches the maximum level in mature adipocytes [4]. In contrast, the expression of the negative regulator, Pref-1, decreases in the process of adipocyte differentiation [5]. Our data indicated that overexpression of miR-378 promoted the differentiation of bovine preadipocytes.

MiRNAs silence gene expression post-transcriptionally through regulating the transcription or translation of target gene mRNAs, thus exerting their biological functions [7]. To analyze the regulatory mechanism of miR-378, we predicted and validated the target genes of miR-378 with bioinformatics and luciferase reporter assays. Our data suggested that E2F2 and RANBP10 are two target genes of miR-378. Overexpression of miR-378 in bovine preadipocytes failed to induce significant changes in the endogenous mRNA expression levels of E2F2 and RANBP10. However, the protein expression levels of E2F2 and RANBP10 were inhibited. These results suggested that miR-378 regulates E2F2 and RANBP10 via inhibiting the translation of E2F2 and RANBP10 mRNAs. E2F2 is a member of the E2F family of transcription factors, and the regulation of E2F transcription factors is rather complex. E2F1-3 are involved in the retinoblastoma protein (Rb)/E2F pathway and exert an regulatory effect on G1-S cell cycle progression [27, 28]. E2F2 binds to Rb and the E2F2/Rb complex inhibits cell cycle progression. In addition, miR-24 can target E2F2 to inhibit cell proliferation [29]. Studies have shown that RANBP10 plays regulatory roles in spindle assembly [30], cell cycle regulation [31], tumor suppression [32], and the reproductive system [32, 33]. Since 
miR-378 targets both E2F2 and RANBP10, two of the important transcription factors closely associated with cell growth, it is reasonable to hypothesize that E2F2 and RANBP10 might involve in the effects of miR-378 on the bovine preadipocyte differentiation.

In summary, we found that miR-378 promoted the differentiation of bovine preadipocytes. E2F2 and RANBP10 were the two target genes of miR-378, and might involve in the effects of miR-378 on the bovine preadipocyte differentiation. The results of the present study provided a basis for further investigation of miRNA-mediated physiological regulation of adipose tissue in bovine.

\section{Acknowledgements}

The authors thank Dr. Xianzhong Yu of Clemson University, Unite States for reading and modifying the manuscript. The present research is supported by the Earmarked Fund for Modern Agro-Industry Technology Research System (CARS-38), National Natural Science Foundation (31201076), China Postdoctoral Science Foundation (2012M520682), Jilin Scientific and Technological Development Foundation (20130522001JH), Key Program for Science and Technology Development of Jilin Province (20140204077NY) in China.

\section{Disclosure Statement}

All the authors have no conflict of interest.

\section{References}

1 Gregoire FM, Smas CM, Sul HS: Understanding adipocyte differentiation. Physiol Rev 1998;78:783-809.

2 Dani C, Smith AG, Dessolin S, Leroy P, Staccini L, Villageois P, Darimont C, Ailhaud G: Differentiation of embryonic stem cells into adipocytes in vitro. J Cell Sci 1997;110:1279-1285.

3 Pittenger MF, Mackay AM, Beck SC, Jaiswal RK, Douglas R, Mosca JD, Moorman MA, Simonetti DW, Craig S, Marshak DR: Multilineage potential of adult human mesenchymal stem cells. Science 1999;284:143-147.

$4 \quad$ Farmer SR: Transcriptional control of adipocyte formation. Cell Metab 2006;4:263-273.

5 Wang Y, Sul HS: Pref-1 regulates mesenchymal cell commitment and differentiation through sox9. Cell Metab 2009;9:287-302.

6 Bartel DP: Micrornas: Genomics, biogenesis, mechanism, and function. Cell 2004;116:281-297.

7 Bartel DP: Micrornas: Target recognition and regulatory functions. Cell 2009;136:215-233.

8 Kim SY, Kim AY, Lee HW, Son YH, Lee GY, Lee JW, Lee YS, Kim JB: Mir-27a is a negative regulator of adipocyte differentiation via suppressing ppargamma expression. Biochem Biophys Res Commun 2010;392:323-328.

9 Kajimoto K, Naraba H, Iwai N: Microrna and 3t3-l1 pre-adipocyte differentiation. RNA 2006;12:1626-1632.

10 Wang Q, Li YC, Wang J, Kong J, Qi Y, Quigg RJ, Li X: Mir-17-92 cluster accelerates adipocyte differentiation by negatively regulating tumor-suppressor rb2/p130. Proc Natl Acad Sci U S A 2008;105:2889-2894.

11 Esau C, Kang X, Peralta E, Hanson E, Marcusson EG, Ravichandran LV, Sun Y, Koo S, Perera RJ, Jain R, Dean NM, Freier SM, Bennett CF, Lollo B, Griffey R: Microrna-143 regulates adipocyte differentiation. J Biol Chem 2004;279:52361-52365.

12 Karbiener M, Fischer C, Nowitsch S, Opriessnig P, Papak C, Ailhaud G, Dani C, Amri EZ, Scheideler M: Microrna mir-27b impairs human adipocyte differentiation and targets ppargamma. Biochem Biophys Res Commun 2009;390:247-251.

13 Sun T, Fu M, Bookout AL, Kliewer SA, Mangelsdorf DJ: Microrna let-7 regulates 3t3-11 adipogenesis. Mol Endocrinol 2009;23:925-931.

14 Trajkovski M, Hausser J, Soutschek J, Bhat B, Akin A, Zavolan M, Heim MH, Stoffel M: Micrornas 103 and 107 regulate insulin sensitivity. Nature 2011;474:649-653.

15 Gerin I, Bommer GT, McCoin CS, Sousa KM, Krishnan V, MacDougald OA: Roles for mirna-378/378* in adipocyte gene expression and lipogenesis. Am J Physiol Endocrinol Metab 2010;299:E198-206. 
16 Gao Y, Li F, Zhang Y, Dai L, Jiang H, Liu H, Zhang S, Chen C, Zhang J: Silencing of adipoq efficiently suppresses preadipocyte differentiation in porcine. Cell Physiol Biochem 2013;31:452-461.

17 Zhang LJ, Zhu YN, Gao Y, Liu SY, Zhai B, Li CH, Liu HY, Chen J, Yuan B, Dai LS, Zhang JB: The mbd4 gene plays an important role in porcine adipocyte differentiation. Cell Physiol Biochem 2014;34:1216-1226.

18 Gagan J, Dey BK, Layer R, Yan Z, Dutta A: Microrna-378 targets the myogenic repressor myor during myoblast differentiation. J Biol Chem 2011;286:19431-19438.

19 Kahai S, Lee SC, Lee DY, Yang J, Li M, Wang CH, Jiang Z, Zhang Y, Peng C, Yang BB: Microrna mir-378 regulates nephronectin expression modulating osteoblast differentiation by targeting galnt-7. PLoS One 2009;4:e7535.

20 Lee DY, Deng Z, Wang CH, Yang BB: Microrna-378 promotes cell survival, tumor growth, and angiogenesis by targeting sufu and fus-1 expression. Proc Natl Acad Sci U S A 2007;104:20350-20355.

21 Bao L, Yan Y, Xu C, Ji W, Shen S, Xu G, Zeng Y, Sun B, Qian H, Chen L, Wu M, Su C, Chen J: Microrna-21 suppresses pten and hsulf-1 expression and promotes hepatocellular carcinoma progression through akt/ erk pathways. Cancer Lett 2013;337:226-236.

22 Liu CT, Zhu ST, Li P, Wang YJ, Zhang H, Zhang ST: Sulf1 inhibits proliferation and invasion of esophageal squamous cell carcinoma cells by decreasing heparin-binding growth factor signaling. Dig Dis Sci 2013;58:1256-1263.

23 Xu S, Linher-Melville K, Yang BB, Wu D, Li J: Micro-rna378 (mir-378) regulates ovarian estradiol production by targeting aromatase. Endocrinology 2011;152:3941-3951.

24 Holst D, Grimaldi PA: New factors in the regulation of adipose differentiation and metabolism. Curr Opin Lipidol 2002;13:241-245.

25 Greenberg AS, McDaniel ML: Identifying the links between obesity, insulin resistance and beta-cell function: Potential role of adipocyte-derived cytokines in the pathogenesis of type 2 diabetes. Eur J Clin Invest 2002;32 Suppl 3:24-34.

26 Jin W, Dodson MV, Moore SS, Basarab JA, Guan LL: Characterization of microrna expression in bovine adipose tissues: A potential regulatory mechanism of subcutaneous adipose tissue development. BMC Mol Biol 2010;11:29.

27 Krek W, Xu G, Livingston DM: Cyclin a-kinase regulation of e2f-1 DNA binding function underlies suppression of an s phase checkpoint. Cell 1995;83:1149-1158.

28 Bartek J, Bartkova J, Lukas J: The retinoblastoma protein pathway and the restriction point. Curr Opin Cell Biol 1996;8:805-814.

29 Lal A, Navarro F, Maher CA, Maliszewski LE, Yan N, O'Day E, Chowdhury D, Dykxhoorn DM, Tsai P, Hofmann O, Becker KG, Gorospe M, Hide W, Lieberman J: Mir-24 inhibits cell proliferation by targeting e2f2, myc, and other cell-cycle genes via binding to "seedless" 3'utr microrna recognition elements. Mol Cell 2009;35:610625.

30 Schulze H, Dose M, Korpal M, Meyer I, Italiano JE, Jr., Shivdasani RA: Ranbp10 is a cytoplasmic guanine nucleotide exchange factor that modulates noncentrosomal microtubules. J Biol Chem 2008;283:1410914119.

31 Hosono K, Noda S, Shimizu A, Nakanishi N, Ohtsubo M, Shimizu N, Minoshima S: Ypel5 protein of the ypel gene family is involved in the cell cycle progression by interacting with two distinct proteins ranbpm and ranbp10. Genomics 2010;96:102-111.

32 Harada N, Yokoyama T, Yamaji R, Nakano Y, Inui H: Ranbp10 acts as a novel coactivator for the androgen receptor. Biochem Biophys Res Commun 2008;368:121-125.

33 Walker CG, Meier S, Mitchell MD, Roche JR, Littlejohn M: Evaluation of real-time pcr endogenous control genes for analysis of gene expression in bovine endometrium. BMC Mol Biol 2009;10:100. 